\title{
Successful Hepatic Arterial Infusion of Chemotherapy in a Patient with Advanced Hepatocellular Carcinoma and Impending Liver Failure
}

\author{
Tsung-Hao Liu a, b Chih-Hung Hsu ${ }^{b, c}$ Yu-Yun Shaob-d \\ a Department of Internal Medicine, National Taiwan University Hospital Hsin-Chu Branch, \\ Hsinchu, Taiwan; ' Department of Oncology, National Taiwan University Hospital, Taipei, \\ Taiwan; ' Graduate Institute of Oncology, National Taiwan University College of Medicine, \\ Taipei, Taiwan; d National Taiwan University Cancer Center, Taipei, Taiwan
}

Dear Editor,

We read with great interest the study of Moriguchi et al. [1] regarding the efficacy of hepatic arterial infusion of chemotherapy (HAIC) in treating advanced hepatocellular carcinoma (HCC) with portal vein tumor thrombosis (PVTT). HAIC has been proposed to be beneficial for patients with advanced HCC owing to a numerically higher response rate than with sorafenib. We would like to share a unique case of a patient with advanced HCC and Child-Pugh (CP) class C liver reserve to endorse the findings of Moriguchi et al. [1].

A 59-year-old male with no prior history of systemic disease, including chronic viral hepatitis or alcoholic liver disease, was found to have an infiltrative mass occupying the majority of the right liver, along with main PVTT and dilated left intrahepatic bile ducts (Fig. 1A, B) identified using computed tomography (CT). Laboratory examination revealed severe hyperbilirubinemia (total/direct bilirubin 36.2/23.6 mg/ $\mathrm{dL})$ and hypoalbuminemia $(2.2 \mathrm{~g} / \mathrm{dL})$, prothrombin time was $13.3 \mathrm{~s}$ (international normalized ratio of 1.26), and the alpha-fetoprotein level was $712.9 \mathrm{ng} / \mathrm{mL}$. Liver reserve was determined to be CP class C. A biopsy of the liver tumor confirmed the diagnosis of HCC. Percutaneous transhepatic cholangiography revealed obstructed left intrahepatic bile ducts for which a biliary drainage tube was inserted. The total bilirubin level decreased to $17.6 \mathrm{mg} / \mathrm{dL}$ but failed to decrease further despite adequate drainage.

Because of the patient's poor liver reserve, sorafenib was not recommended. The patient consented to receive HAIC and was given $500 \mathrm{mg} / \mathrm{m}^{2} /$ day of 5 -fluorouracil on days $1-3$ and $60 \mathrm{mg} / \mathrm{m}^{2}$ of cisplatin on day 2, which was repeated every 3 weeks [2]. The total bilirubin level decreased to $5.3 \mathrm{mg} / \mathrm{dL}$ before the second HAIC cycle and then further improved to $1.5 \mathrm{mg} / \mathrm{dL}$ before the third cycle (Fig. 2). The alpha-fetoprotein level also decreased to $183.4 \mathrm{ng} / \mathrm{mL}$. After 3 cycles of HAIC, a CT scan showed right liver tumor shrinkage and resolution of the main and left PVTT (Fig. 1C, D). Percutaneous transhepatic cholangiog-

Yu-Yun Shao, MD

Department of Oncology, National Taiwan University Hospital

No. 7, Chung-Shan South Road

Taipei 10002 (Taiwan)

E-Mail yuyunshao@gmail.com 


\section{Liver Cancer}

Fig. 1. Computed tomography scans before hepatic arterial infusion of chemotherapy (HAIC) treatment (A, B), after 3 HAIC cycles (C, D), and after 12 HAIC cycles $(\mathbf{E}, \mathbf{F})$.

\begin{tabular}{l|l}
\hline Liver Cancer \\
\hline DOI: 10.1159/000486763 & $\begin{array}{l}\text { (c) 2018 S. Karger AG, Basel } \\
\text { www.karger.com/lic }\end{array}$ \\
\hline
\end{tabular}

Liu et al.: Successful Hepatic Arterial Infusion of Chemotherapy in a Patient with Advanced Hepatocellular Carcinoma and Impending Liver Failure
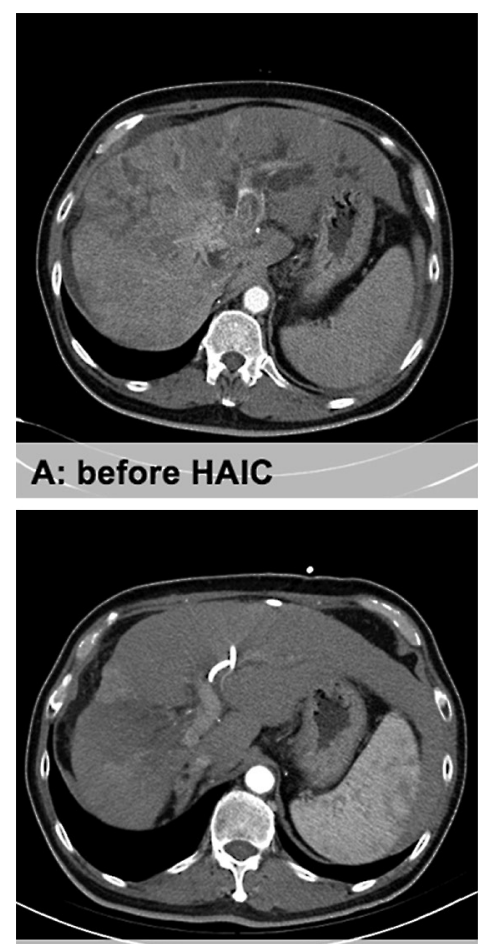

C: after 3 cycles of HAIC

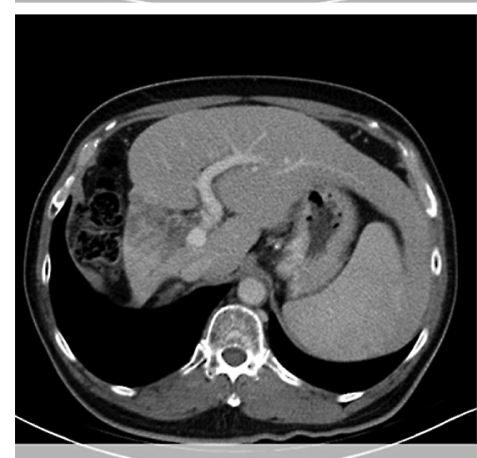

E: after 12 cycles of HAIC

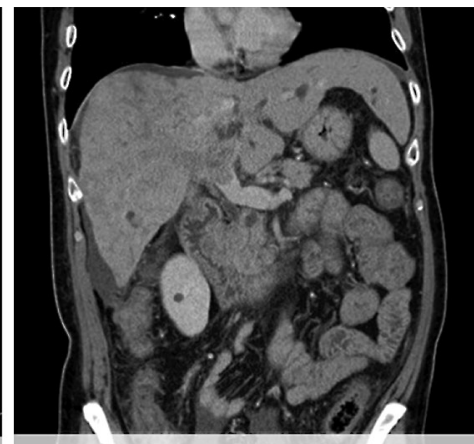

\section{B: before HAIC}

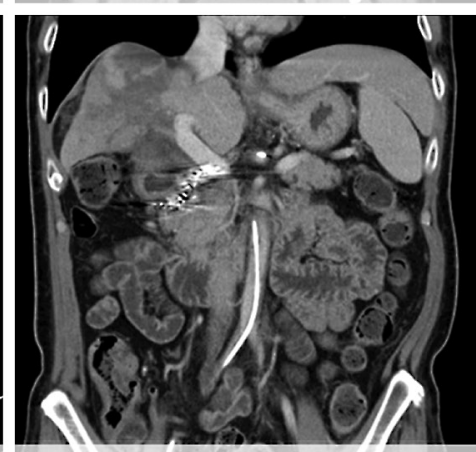

D: after 3 cycles of HAIC

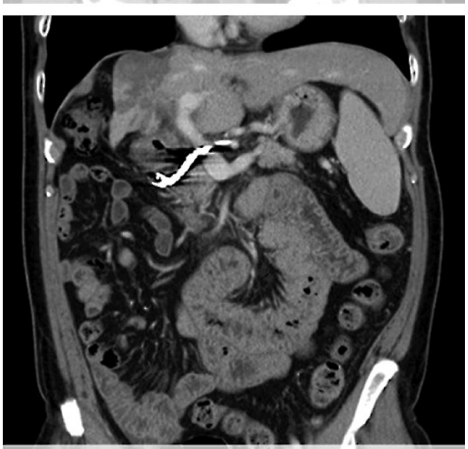

F: after 12 cycles of HAIC

raphy revealed biliary obstruction resolution; therefore, the drainage tube was removed. The total bilirubin level stabilized to approximately $1 \mathrm{mg} / \mathrm{dL}$, and the albumin level increased to $4 \mathrm{~g} / \mathrm{dL}$. The patient's liver reserve was thus reclassified as CP class A (Fig. 2). All HAIC cycles were well tolerated with no grade 3 or 4 toxicities. The most severe adverse effect was a grade 2 thrombocytopenia that did not require a dose adjustment. A serial follow-up CT scan after 12 cycles of HAIC showed a sustained positive response to HAIC (Fig. 1E, F).

New lung metastases were identified 6 months later, and HAIC was discontinued. At that time, a total of 19 HAIC cycles were given within 18 months. Upon disease progression, the patient remained in CP class A liver reserve; therefore, sorafenib was given as subsequent treatment. Four months after the new metastases had been diagnosed, the patient continued to be treated with sorafenib and has now survived more than 2 years since the initial diagnosis of advanced HCC.

This case demonstrated the potential of using HAIC in patients with advanced HCC and poor liver reserve. HAIC might provide a higher response rate than sorafenib, although no randomized studies have been conducted [3]. This patient's liver reserve improved substantially from CP class C to A during the HAIC treatment course. Consequently, treatment with sorafenib was possible after the disease progressed 


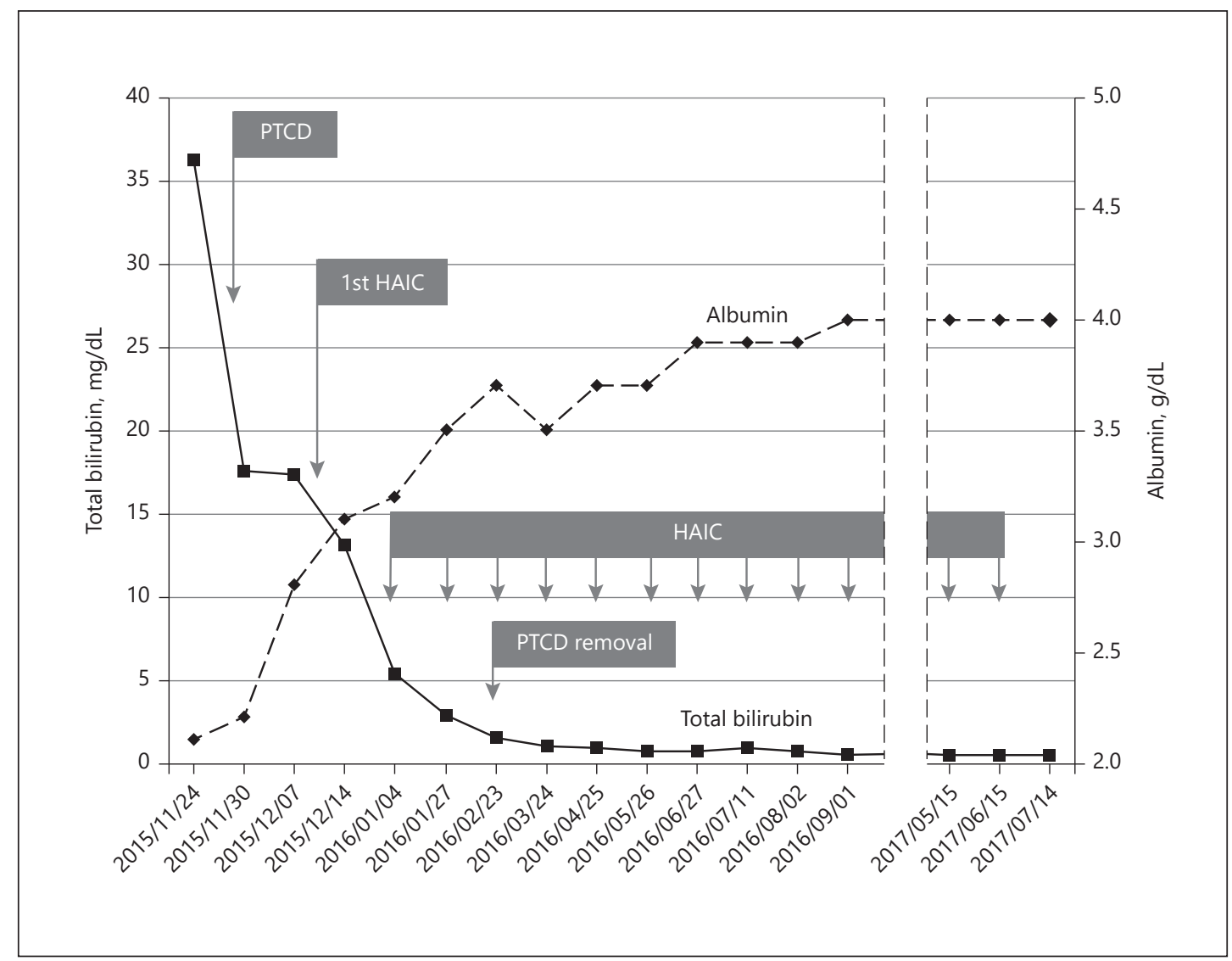

Fig. 2. Serial changes in serum total bilirubin and albumin levels after the initiation of hepatic arterial infusion of chemotherapy (HAIC). PTCD, percutaneous transhepatic cholangial drainage.

on HAIC treatment. This patient survived for more than 2 years, which is much longer than the reported median survival of patients with advanced HCC and CP class C liver reserve (1.5-4.0 months) [4].

We did not choose targeted agents, such as sorafenib, or immunotherapy as a first-line therapy for this patient. Most targeted agents have benefits for patients with advanced HCC through disease stabilization rather than tumor shrinkage. Immunotherapy with checkpoint inhibitors, such as nivolumab, might provide a higher response rate (20\%), but the median time to response is 4.1 months [5]. Because our patient had a considerably large liver tumor with impending hepatic failure, disease stabilization or a slow tumor response might not have been ideal. Therefore, we considered HAIC an attractive treatment option.

Approved therapy is not available for patients with advanced HCC and CP class B or C liver reserves. Among patients who received sorafenib, the median survival was approximately 5.2 and 2.6 months for patients with CP class B and C liver reserve, respectively [4]. HAIC has been reported to provide a numerically comparable median survival in patients with CP class B or C liver reserve [6, 7]. Adverse effects from HAIC treatment are not significantly worse in patients with CP class B and C liver reserve than in patients with CP class A [6]. Device issues were infrequent with experienced physicians and institutes. HAIC may serve as a viable treatment option in selected patients with CP class B or C liver reserve.

In conclusion, we report the success of HAIC in a patient with advanced HCC and impending liver failure. The treatment resulted in a dramatic tumor response, an improved liver reserve, and prolonged survival. 
Liu et al.: Successful Hepatic Arterial Infusion of Chemotherapy in a Patient with Advanced Hepatocellular Carcinoma and Impending Liver Failure

\section{References}

1 Moriguchi M, Aramaki T, Nishiofuku H, Sato R, Asakura K, Yamaguchi K, et al: Sorafenib versus hepatic arterial infusion chemotherapy as initial treatment for hepatocellular carcinoma with advanced portal vein tumor thrombosis. Liver Cancer 2017;6:275-286.

$\checkmark 2$ Shao Y-Y, Liang P-C, Wu Y-M, Huang C-C, Huang K-W, Cheng JC, et al: A pilot study of hepatic arterial infusion of chemotherapy for patients with advanced hepatocellular carcinoma who have failed anti-angiogenic therapy. Liver Int 2013;33:1413-1419.

3 Nouso K, Miyahara K, Uchida D, Kuwaki K, Izumi N, Omata M, et al: Effect of hepatic arterial infusion chemotherapy of 5-fluorouracil and cisplatin for advanced hepatocellular carcinoma in the Nationwide Survey of Primary Liver Cancer in Japan. Br J Cancer 2013;109:1904-1907.

-4 Marrero JA, Kudo M, Venook AP, Ye S-L, Bronowicki J-P, Chen X-P, et al: Observational registry of sorafenib use in clinical practice across Child-Pugh subgroups: The GIDEON study. J Hepatol 2016;65:1140-1147.

$>5$ El-Khoueiry AB, Sangro B, Yau T, Crocenzi TS, Kudo M, Hsu C, et al: Nivolumab in patients with advanced hepatocellular carcinoma (CheckMate 040): an open-label, non-comparative, phase 1/2 dose escalation and expansion trial. Lancet 2017;389:2492-2502.

-6 Miyaki D, Aikata H, Honda Y, Naeshiro N, Nakahara T, Tanaka M, et al: Hepatic arterial infusion chemotherapy for advanced hepatocellular carcinoma according to Child-Pugh classification. J Gastroenterol Hepatol 2012; 27:1850-1857.

7 Kudo M, Osaki Y, Matsunaga T, Kasugai H, Oka H, Seki T: Hepatocellular carcinoma in Child-Pugh C cirrhosis: prognostic factors and survival benefit of nontransplant treatments. Dig Dis 2013;31:490-498. 